\title{
Derivation of a fundamental diagram for urban traffic flow
}

\section{Journal Article}

Author(s):

Helbing, Dirk

Publication date:

2009

Permanent link:

https://doi.org/10.3929/ethz-b-000157287

Rights / license:

In Copyright - Non-Commercial Use Permitted

Originally published in:

The European Physical Journal B 70(2), https://doi.org/10.1140/epjb/e2009-00093-7 


\title{
Derivation of a fundamental diagram for urban traffic flow
}

\author{
D. Helbing ${ }^{\mathrm{a}}$ \\ ETH Zurich, UNO D11, Universitätstr. 41, 8092 Zurich, Switzerland
}

Received 11 July 2008 / Received in final form 22 December 2008

Published online 14 March 2009 - (C) EDP Sciences, Società Italiana di Fisica, Springer-Verlag 2009

\begin{abstract}
Despite the importance of urban traffic flows, there are only a few theoretical approaches to determine fundamental relationships between macroscopic traffic variables such as the traffic density, the utilization, the average velocity, and the travel time. In the past, empirical measurements have primarily been described by fit curves. Here, we derive expected fundamental relationships from a model of traffic flows at intersections, which suggest that the recently measured fundamental diagrams for urban flows can be systematically understood. In particular, this allows one to derive the average travel time and the average vehicle speed as a function of the utilization and/or the average number of delayed vehicles.
\end{abstract}

PACS. 89.40.Bb Land transportation - 47.10.ab Conservation laws and constitutive relations $-51.10 .+y$ Kinetic and transport theory of gases

\section{Introduction}

The study of urban traffic flows has a long history (see Ref. [1] for an overview). For more than a decade now, physicists have contributed various interesting models, ranging from cellular automata [2-4] to fluid-dynamic approaches $[5,6]$. Complementary, one should mention, for example, reference $[7,8]$ as representatives of publications by traffic engineers, and also continuous microscopic flow models used in commercial software tools such as VISSIM.

One research area in traffic physics is the transition from free to congested traffic in urban road networks $[9,10]$, which started off with the paper by Biham et al. [11]. Interestingly enough, the spreading of congestion seems to share some features with cascading failures $[12]$.

In the following, we will focus on the study of fundamental relationships between flow, utilization, and density on the one hand and the average velocity or travel time on the other hand. Such relationships were already studied in the 60ies [13-23], but this work primarily took a phenomenological approach. Moreover, the recorded data in the regime of congested road networks scattered enormously. Thus, it was hard to fit a curve. Consequently, many different relationships were proposed. The probably most wide-spread formula is the one published by the Bureau of Public Roads [16]. Accordingly, the travel time $\mathcal{T}_{i}$ of an urban road section $i$ with capacity $C_{i}$ would follow the capacity constraint function

$$
\mathcal{T}_{i}=\mathcal{T}_{i}^{0}\left[1+\alpha_{i}\left(\frac{A_{i}}{C_{i}}\right)^{\beta_{i}}\right] .
$$

\footnotetext{
a e-mail: dhelbing@ethz.ch
}

(see Fig. 5). Here, $A_{i}$ is the arrival flow in that road section and $\mathcal{T}_{i}^{0}$ the travel time for light traffic conditions, while $\alpha_{i} \approx 0.5$ and $\beta_{i} \approx 4$ are fit parameters. It is obvious that this formula does not diverge when the capacity $C_{i}$ is reached, as one may expect. This is just one of the many theoretical inconsistencies of the proposed phenomenological formulas. It is no wonder that the subject of fundamental diagrams for urban traffic has recently been taken up again [24-31].

The first measurement of an urban fundamental diagram was presented by Godfrey [32]. Ten years later, even Robert Herman and nobel prize laureate Ilya Prigogine addressed traffic flow in cities $[33,34]$. They tried to derive fundamental relationships via a statistical physics approach, which however still contained phenomenological elements. Their "two-fluid approach" considered moving and standing traffic and the mutual interdependencies between them.

Recently, the issue of urban gridlock was reanimated by Carlos Daganzo [28]. Together with Geroliminis, he presented convincing evidence for a fundamental diagram of urban traffic flow $[29,30]$. Before, fundamental diagrams were mainly used for the study of freeway systems [36]. There, they were very useful to understand capacity effects [1], different traffic states [37], and the traffic dynamics, in particularly the propagation of shock fronts [38]. It is, therefore, not surprising that people are eager to find fundamental relationships for urban traffic as well.

The most recent progress is an approximation of the urban fundamental diagram by Daganzo and Geroliminis, which is based on cutting away parts of the flow-density plane [31]. It appears possible to construct a relationship with kinematic wave theory [35]. In contrast to the 
density-based approach by Daganzo et al., we will pursue an alternative, utilization-based approach. This is common in queueing theory [39] and transportation planning, where formulas such as the capacity constraint function (1) are used.

After discussing elementary relationships for cyclically signalized intersections of urban road networks in Section 2, we will start in Section 3 with the discussion of undersaturated traffic conditions and derive a relationship for the average travel time as a function of the utilization or the number of delayed vehicles. Furthermore, we will determine a formula for the average speed. In Section 4, we will extend the analysis to congested road conditions, where the intersection capacity is exceeded. Afterwards, in Section 5, we will indicate how to deal with oversaturated networks, where the link capacity is insufficient to take up all vehicles that would like to enter a road section. Finally, Section 6 provides a summary and discussion. In particularly, we will address issues regarding the transfer of link-based fundamental diagrams to urban areas. We also try to connect the density-based fundamental diagram of Daganzo et al. with the utilization-based approach developed here.

\section{Elementary relationships for cyclically operated intersections}

Let us study a single intersection with a periodically operated traffic light. We shall have green phases $j$ of duration $\Delta T_{j}$, during which one or several of the traffic streams $i$ are served. $\beta_{i j}$ shall be 1 , if traffic stream $i$ is served by green phase $j$, otherwise $\beta_{i j}=0$. The setup time after phase $j$ shall require a time period $\tau_{j}$. It may be imagined to correspond to the time period of the amber light (although in practice, this has to be corrected for reaction times and intersection clearing times). The sum of setup times will be called the "lost service time"

$$
T_{\mathrm{los}}=\sum_{j} \tau_{j}
$$

while the sum of all green time periods and setup times will be called the "cycle time"

$$
T_{\mathrm{cyc}}=\sum_{j}\left(\Delta T_{j}+\tau_{j}\right)=T_{\mathrm{los}}+\sum_{j} \Delta T_{j}
$$

The green times are also sometimes expressed as fractions $f_{j} \geq 0$ of the cycle time, i.e.

$$
\Delta T_{j}=f_{j} T_{\mathrm{cyc}}
$$

with $\sum_{j} f_{j}<1$. After inserting this into equation (3) and rearranging terms, we get

$$
T_{\mathrm{cyc}}\left(\left\{f_{j}\right\}\right)=\frac{T_{\mathrm{los}}}{1-\sum_{j} f_{j}},
$$

i.e. the cycle time is proportional to the lost service time $T_{\text {los }}$.

Assuming average inflows $A_{i}$ per lane, the number of vehicles belonging to traffic stream $i$, which must be served within one cycle time $T_{\text {cyc }}$, amounts to $A_{i} T_{\text {cyc. }}$. In order to avoid the formation of growing queues (i.e. the onset of congestion), the number of served vehicles per cycle time and lane must reach this value. The number of vehicles of traffic stream $i$ potentially served during the green phases $\Delta T_{j}$ is given by $\sum_{j} Q_{i j} \beta_{i j} \Delta T_{j}$ per lane, where $Q_{i j}$ denotes the outflow capacity (discharge flow) per lane, when stream $i$ is served by phase $j$. If we want to have a certain amount of excess capacity to cope with a variability of the inflows, we may demand

$$
\left(1+\delta_{i}\right) A_{i} T_{\text {cyc }}=\sum_{j} Q_{i j} \beta_{i j} \Delta T_{j}=\sum_{j} Q_{i j} \beta_{i j} f_{j} T_{\text {cyc }}
$$

with $\delta_{i}>0$. This linear set of equations may be solved for the green time fractions $f_{j}$. In the following, we will assume the case, where not several traffic streams $i$ are served in parallel by one and the same green phase, but where each phase $j$ serves a single traffic stream $i$. Then, we may assume $\beta_{i j}=1$, if $j=i$, and $\beta_{i j}=0$ otherwise. Furthermore, $Q_{i j}=Q_{i i}=\widehat{Q}_{i}$. With this, equation (6) implies

$$
f_{i}\left(u_{i}, \delta_{i}\right)=\left(1+\delta_{i}\right) \frac{A_{i}}{\widehat{Q}_{i}}=\left(1+\delta_{i}\right) u_{i}
$$

where

$$
u_{i}=\frac{A_{i}}{\widehat{Q}_{i}}
$$

is called the utilization of the service or outflow capacity $\widehat{Q}_{i}$ (see Fig. 1). $\delta_{i} \geq 0$ is a safety factor to cope with variations in the arrival flow (inflow) $A_{i}$. According to equation (5) we must have

$$
0<1-\sum_{i} f_{i}=1-\sum_{i}\left(1+\delta_{i}\right) \frac{A_{i}}{\widehat{Q}_{i}}
$$

Otherwise, we will have growing vehicle queues from one signal cycle to the next, corresponding to the congested traffic regime.

Note that, at time $t$, the number $N_{i}(t)$ of vehicles per lane in the road section reserved for traffic stream $i$ is given by the time integral of the arrival flow $A_{i}(t)$ minus the departure flow $\gamma_{i}(t) O_{i}(t)$ :

$$
N_{i}(t)=\int_{t_{0}}^{t} d t^{\prime}\left[A_{i}\left(t^{\prime}\right)-\gamma_{i}\left(t^{\prime}\right) O_{i}\left(t^{\prime}\right)\right]
$$

Here, the starting time $t_{0}$ must be properly chosen to give the correct initial number of vehicles on road section $i$. During the amber and red time periods, we set the permeability $\gamma_{i}(t)=0$, as there is no outflow, while $\gamma_{i}(t)=1$ during green phases. The departure flow $O_{i}$ per lane is given by the service capacity $\widehat{Q}_{i}$ per lane, as long as there 


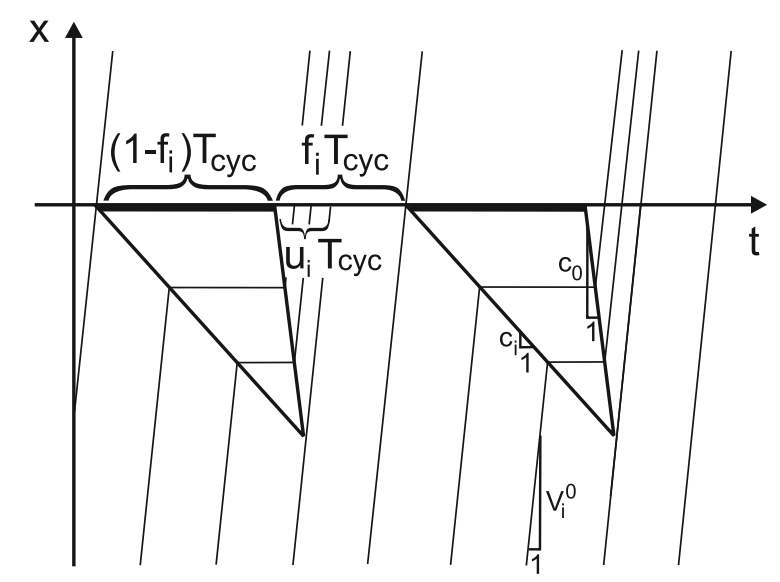

Fig. 1. Schematic illustration of vehicle trajectories for a traffic light, which has an amber and red time period of altogether $\left(1-f_{i}\right) T_{\text {cyc }}$ and a green time period of $f_{i} T_{\text {cyc }}$. Vehicles move forward at the free speed $V_{i}^{0}$ or are stopped in a vehicle queue (horizontal lines), which forms during the amber and red time period behind the traffic light (located at the $t$-axis). The speed of the upstream moving congestion front is given by the arrival flow [38] and denoted by $c_{i} \leq 0$. The dissolution speed $c_{0}<0$ of congested traffic is a characteristic constant with $\left|c_{0}\right| \geq\left|c_{i}\right|[6,40]$. The average delay time can be determined by averaging over the waiting times in the triangular areas. Note that for the case of an excess greentime $\left(f_{i}>u_{i}\right)$, vehicles may pass the traffic light without any delay.

is a positive number $\Delta N_{i}(t)>0$ of delayed vehicles. If $Q_{\text {out }}$ represents the characteristic outflow from congested traffic per lane into an area of free flow, the overall service capacity by all service lanes is given by the minimum of the number of lanes $I_{i}$ used by vehicle stream $i$ upstream the intersection, and the number $I_{i}^{\prime}$ of lanes downstream of it:

$$
I_{i} \widehat{Q}_{i}=\min \left(I_{i}, I_{i}^{\prime}\right) Q_{\text {out }}, \quad \text { i.e. } \quad \widehat{Q}_{i}=\min \left(1, \frac{I_{i}^{\prime}}{I_{i}}\right) Q_{\text {out }} .
$$

When the vehicle queue forming behind a traffic light has completely resolved, the outflow from the road section used by vehicle stream $i$ drops from $\widehat{Q}_{i}$ to a lower value. Then, if the greentime period continues, the outflow $O_{i}(t)$ per lane corresponds to the arrival flow $A_{i}\left(t-\mathcal{T}_{i}^{0}\right)$ per lane expected at the end of road section $i$ under free flow conditions [40]. Here, $\mathcal{T}_{i}^{0}=L_{i} / V_{i}^{0}$ represents the travel time under free flow conditions, which is obtained by division of the length $L_{i}$ of the road section reserved for stream $i$ by the free speed $V_{i}^{0}$. Considering also the above definition of the permeabilities $\gamma_{i}(t)$ reflecting the time-dependent states of the traffic signal, we have

$$
\gamma_{i}(t) O_{i}(t)=\gamma_{i}(t) \begin{cases}\widehat{Q}_{i} & \text { if } \Delta N_{i}(t)>0 \\ A_{i}\left(t-\mathcal{T}_{i}^{0}\right) & \text { otherwise. }\end{cases}
$$

The number $\Delta N_{i}(t)$ of delayed vehicles per lane at time $t$ on the road section reserved for vehicle stream $i$ can be easily determined as well. In contrast to equation (10), we have to subtract the integral of the departure flow from the integral of the arrival flow $A_{i}\left(t-\mathcal{T}_{i}^{0}\right)$ expected at the $e n d$ of road section $i$ under free flow conditions. Altogether, the number of delayed vehicles can be calculated as

$$
\Delta N_{i}(t)=\int_{t_{0}}^{t} d t^{\prime}\left[A_{i}\left(t^{\prime}-\mathcal{T}_{i}^{0}\right)-\gamma_{i}\left(t^{\prime}\right) O_{i}\left(t^{\prime}\right)\right] .
$$

If the traffic flow is organized as a vehicle platoon and the green phase is synchronized with its arrival at the traffic light, the number of delayed vehicles is zero. However, if the traffic flow $A_{i}$ is uniform, we find

$$
\Delta N_{i}(t)=A_{i} \cdot\left(t-t_{0}\right)-\int_{t_{0}}^{t} d t^{\prime} \gamma_{i}\left(t^{\prime}\right) O_{i}\left(t^{\prime}\right)
$$

Let us assume that $t_{0}$ denotes the time when the green phase for traffic stream $i$ ended. Then, the next green phase for this traffic stream starts at time $t_{0}^{\prime}=t_{0}+(1-$ $\left.f_{i}\right) T_{\text {cyc }}$, as $f_{i} T_{\text {cyc }}$ is the green time period and $\left(1-f_{i}\right) T_{\text {cyc }}$ amounts to the sum of the amber and red time periods. Due to $O_{i}(t) \geq A_{i}(t)$ and $O_{i}\left(t_{0}^{\prime}\right)>A_{i}\left(t_{0}^{\prime}\right), t_{0}^{\prime}-t_{0}=(1-$ $\left.f_{i}\right) T_{\text {cyc }}$ is also the time period after which the maximum number $\Delta N_{i}^{\max }$ of delayed vehicles is reached.

In case of a uniform arrival of vehicles at the rate $A_{i}=$ $u_{i} \widehat{Q}_{i}$ per lane we have

$$
\begin{aligned}
\Delta N_{i}^{\max }\left(u_{i},\left\{f_{j}\right\}\right) & =A_{i}\left(1-f_{i}\right) T_{\text {сус }}\left(f_{i}\right) \\
& =u_{i} \widehat{Q}_{i}\left(1-f_{i}\right) T_{\text {cyc }}\left(\left\{f_{j}\right\}\right) .
\end{aligned}
$$

Since $\widehat{Q}_{i}-A_{i}$ is the rate at which this vehicle queue can be reduced (considering the further uniform arrival of vehicles at rate $A_{i}$ ), it takes a green time period of

$$
T_{i}\left(u_{i},\left\{f_{j}\right\}\right)=\frac{A_{i}\left(1-f_{i}\right) T_{\mathrm{cyc}}}{\widehat{Q}_{i}-A_{i}}=\frac{u_{i}\left(1-f_{i}\right)}{1-u_{i}} T_{\mathrm{cyc}}\left(\left\{f_{j}\right\}\right),
$$

until this vehicle queue is again fully resolved, and newly arriving vehicles can pass the traffic light without any delay.

Finally, let us determine the average delay time of vehicles. If we have a platoon of vehicles which is served by a properly synchronized traffic light, the average delay is $\mathcal{T}_{i}^{\text {av }}=\mathcal{T}_{i}^{\text {min }}=0$. However, if we have a constant arrival flow $A_{i}$, the average delay $\mathcal{T}_{i}^{\text {av }}$ of queued vehicles is given by the arithmetic mean $\left(\mathcal{T}_{i}^{\text {max }}+\mathcal{T}_{i}^{\text {min }}\right) / 2$ of the maximum delay for the first vehicle in the queue behind the traffic light, which corresponds to the amber plus red time period

$$
\mathcal{T}_{i}^{\max }\left(\left\{f_{j}\right\}\right)=\left(1-f_{i}\right) T_{\text {cyc }}\left(\left\{f_{j}\right\}\right),
$$

and the minimum delay $\mathcal{T}_{i}^{\text {min }}=0$ of a vehicle arriving just at the time when the queue is fully dissolved. To get the average delay, we have to weight this by the percentage of delayed vehicles. While the number of vehicles arriving during the cycle time $T_{\text {cyc }}$ is $A_{i} T_{\text {cyc }}$, the number of undelayed vehicles is given by $A_{i}\left(\Delta T_{i}-T_{i}\right)$. Considering formulas (4) and (16), the excess green time is

$$
\Delta T_{i}-T_{i}=f_{i} T_{\mathrm{cyc}}-\frac{u_{i}\left(1-f_{i}\right) T_{\mathrm{cyc}}}{1-u_{i}}=\frac{f_{i}-u_{i}}{1-u_{i}} T_{\mathrm{cyc}} .
$$




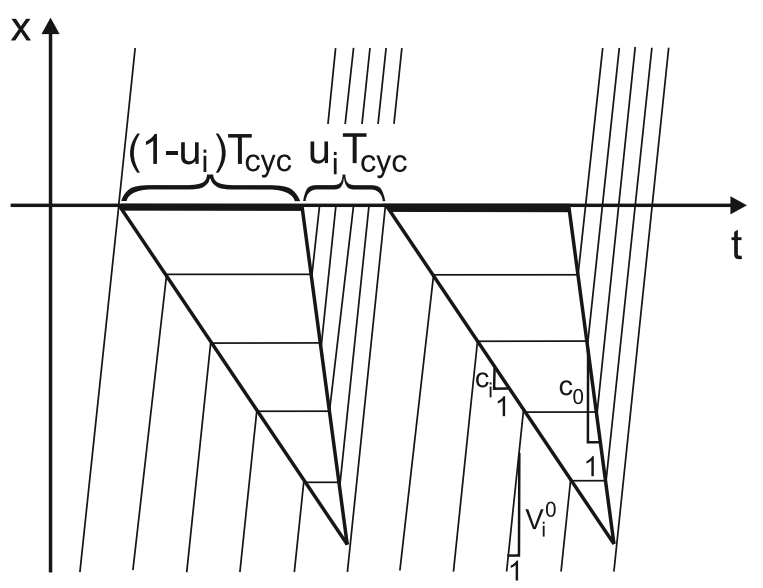

Fig. 2. Schematic illustration of vehicle trajectories and signal operation in the case $f_{i}=u_{i}$, where there are no excess green times so that the traffic light is turned red as soon as the vehicle queue has been fully resolved.

Hence, the percentage of delayed vehicles is

$$
\frac{A_{i}\left[T_{\mathrm{cyc}}-\left(\Delta T_{i}-T_{i}\right)\right]}{A_{i} T_{\mathrm{cyc}}}=1-\frac{f_{i}-u_{i}}{1-u_{i}}=\frac{1-f_{i}}{1-u_{i}} \leq 1 .
$$

Altogether, the average delay of all vehicles is expected to be

$$
\begin{aligned}
\mathcal{T}_{i}^{\mathrm{av}}\left(u_{i},\left\{f_{j}\right\}\right) & =\frac{\left(1-f_{i}\right)}{\left(1-u_{i}\right)} \frac{\mathcal{T}_{i}^{\max }\left(\left\{f_{j}\right\}\right)}{2} \\
& =\frac{\left(1-f_{i}\right)^{2}}{1-u_{i}} \frac{T_{\mathrm{cyc}}\left(\left\{f_{j}\right\}\right)}{2} .
\end{aligned}
$$

Inserting equation (5) gives

$$
\mathcal{T}_{i}^{\text {av }}\left(u_{i},\left\{f_{j}\right\}\right)=\frac{\left(1-f_{i}\right)^{2}}{\left(1-u_{i}\right)} \frac{T_{\text {los }}}{2\left(1-\sum_{j} f_{j}\right)},
$$

while with equation (15) we obtain

$$
\mathcal{T}_{i}^{\text {av }}\left(u_{i},\left\{f_{j}\right\}\right)=\frac{1-f_{i}}{1-u_{i}} \frac{\Delta N_{i}^{\max }\left(u_{i},\left\{f_{j}\right\}\right)}{2 u_{i} \widehat{Q}_{i}} .
$$

Therefore, the average delay time is proportional to the maximum queue length $\Delta N_{i}^{\max }$, but the prefactor depends on $f_{i}=\left(1+\delta_{i}\right) u_{i}$ (or the safety factor $\delta_{i}$, respectively). In case of no excess green time $\left(\delta_{i}=0\right.$ and $f_{i}=u_{i}$ ), we just have

$$
\begin{aligned}
\mathcal{T}_{i}^{\mathrm{av}}\left(\left\{u_{j}\right\}\right) & =\left(1-u_{i}\right) \frac{T_{\mathrm{cyc}}\left(\left\{u_{j}\right\}\right)}{2}=\frac{\Delta N_{i}^{\max }\left(\left\{u_{j}\right\}\right)}{2 A_{i}} \\
& =\frac{\Delta N_{i}^{\max }\left(\left\{u_{j}\right\}\right)}{2 u_{i} \widehat{Q}_{i}}
\end{aligned}
$$

(see Fig. 2).

Similarly to the average travel time, we may determine the average queue length. As the average number of delayed vehicles is $\left(\Delta N_{i}^{\max }+0\right) / 2$ and a fraction $\left(1-f_{i}\right) /\left(1-u_{i}\right) \leq 1$ of vehicles is delayed, together with equations (15) and (20) we find

$$
\begin{aligned}
\Delta N_{i}^{\mathrm{av}}\left(u_{i},\left\{f_{j}\right\}\right) & =\frac{\left(1-f_{i}\right)}{\left(1-u_{i}\right)} \frac{\Delta N_{i}^{\max }\left(u_{i},\left\{f_{j}\right\}\right)}{2} \\
& =u_{i} \widehat{Q}_{i} \frac{\left(1-f_{i}\right)^{2}}{\left(1-u_{i}\right)} \frac{T_{\text {cyc }}\left(\left\{f_{j}\right\}\right)}{2} \\
& =u_{i} \widehat{Q}_{i} \mathcal{T}_{i}{ }^{\mathrm{av}}\left(u_{i},\left\{f_{j}\right\}\right) .
\end{aligned}
$$

This remarkably simple relationship is known in queuing theory as Little's Law [39], which holds for time-averaged variables even in the case of non-uniform arrivals, if the system behaves stable (i.e. the queue length is not systematically growing or shrinking). It also allows one to establish a direct relationship between the "average vehicle density"

$$
\rho_{i}^{\mathrm{av}}=\frac{\Delta N_{i}^{\mathrm{av}}}{L_{i}}
$$

on the road section used by stream $i$ and the utilization $u_{i}=A_{i} / \widehat{Q}_{i}$, namely

$$
\rho_{i}^{\mathrm{av}}=\frac{u_{i} \widehat{Q}_{i} \mathcal{T}_{i}^{\mathrm{av}}}{L_{i}} .
$$

Note that the average density $\rho_{i}^{\text {av }}$ has the same dependencies on other variables as $\Delta N_{i}^{\text {av }}$ or $\mathcal{T}_{i}^{\text {av }}$, and an additional dependency on $L_{i}$, i.e. the more natural quantity to use is the queue length $\Delta N_{i}^{\text {av }}$.

\subsection{Efficiency of traffic operation}

In reality, the average delay time will depend on the timedependence of the inflow $A_{i}(t)$, and on how well the traffic light is coordinated with the arrival of vehicle platoons. In particular, this implies a dependence on the signal offsets. In the best case, the average delay is zero, but in the worst case, it may also be larger than

$$
\frac{1-u_{i}}{2} T_{\mathrm{cyc}}\left(\left\{u_{j}\right\}\right)=\frac{\left(1-u_{i}\right) T_{\mathrm{los}}}{2\left(1-\sum_{j} u_{j}\right)}
$$

see equation (20) with $f_{i}=u_{i}$. We may, therefore, introduce an efficiency coefficient $\epsilon_{i}$ by the definition

$$
\mathcal{T}_{i}^{\mathrm{av}}\left(\left\{u_{j}\right\}, \epsilon_{i}\right)=\left(1-\epsilon_{i}\right) \frac{\left(1-u_{i}\right) T_{\mathrm{los}}}{2\left(1-\sum_{j} u_{j}\right)}
$$

or, considering equation (24), equivalently by

$$
\Delta N_{i}^{\mathrm{av}}\left(\left\{u_{j}\right\}, \epsilon_{i}\right)=\left(1-\epsilon_{i}\right) u_{i} \widehat{Q}_{i} \frac{\left(1-u_{i}\right) T_{\mathrm{los}}}{2\left(1-\sum_{j} u_{j}\right)} .
$$

For $\epsilon_{i}=1$, the traffic light is perfectly synchronized with platoons in traffic stream $i$, i.e. vehicles are served without any delay, while for $\epsilon_{i}=0$, the delay corresponds to uniform arrivals of vehicles, when no excess green time is given. If the traffic light is not well synchronized with the arrival of vehicle platoons, we may even have $\epsilon_{i}<0$. 
It also makes sense to define an efficiencies not only for the traffic phases, but also for the operation of a traffic light (i.e. the full cycle). This can be done by averaging over all efficiencies $\epsilon_{i}$ (and potentially weighting them by the number $u_{i} \widehat{Q}_{i} T_{\text {cyc }}$ of vehicles arriving in one cycle. Therefore, it makes sense to define the intersection efficiency as

$$
\epsilon=\frac{\sum_{i} \epsilon_{i} u_{i} \widehat{Q}_{i}}{\sum_{i} u_{i} \widehat{Q}_{i}}
$$

Note that, particularly in cases of pulsed rather than uniform arrivals of vehicles, the efficiencies $\epsilon_{i}$ depend on the cycle time $T_{\text {cyc }}$ and, therefore, also on the utilizations $u_{i}$. Increasing the efficiency $\epsilon_{i}$ for one traffic stream $i$ will often (but not generally) reduce the efficiency $\epsilon_{j}$ of another traffic stream $j$, which poses a great challenge to traffic optimization.

The exact value of the efficiency $\epsilon_{i}$ depends on many details such as the time-dependence of the arrival flow $A_{i}(t)$ and its average value $\bar{A}_{i}$, the length $L_{i}$ of the road section, and the signal control scheme (fixed cycle time or not, adaptive green phases or not, signal offsets, etc.). These data and the exact signal settings are often not fully available and, therefore, it is reasonable to consider $\epsilon_{i}$ as fit parameters rather than deriving complicated formulas for them. Nevertheless, we will demonstrate the general dependence on the utilization $u_{i}$ in the following.

For this, we will study the case of excess green times $\left(\delta_{i}>0\right)$, which are usually chosen to cope with the stochasticity of vehicle arrivals, i.e. the fact that the number of vehicles arriving during one cycle time is usually fluctuating. The choice $\delta_{i}>0$, i.e. $f_{i}>u_{i}$, also implies

$$
\frac{A_{i} T_{\mathrm{cyc}}}{T_{\mathrm{cyc}}}=u_{i} \widehat{Q}_{i}<f_{i} \widehat{Q}_{i}=\frac{\Delta T_{i}}{T_{\mathrm{cyc}}} \widehat{Q}_{i} .
$$

This reflects the well-known observation that the average departure flow $A_{i}=u_{i} \widehat{Q}_{i}$ usually does not reach the value given by the green time fraction $f_{i}$ times the saturation flow $\widehat{Q}_{i}$.

The efficiency $\epsilon_{i}$ related with a value $\delta_{i}>0$ may be derived from equations (21) and (28). We obtain

$$
1-\epsilon_{i}=\frac{\left(1-f_{i}\right)^{2}}{\left(1-u_{i}\right)^{2}} \frac{\left(1-\sum_{j} u_{j}\right)}{\left(1-\sum_{j} f_{j}\right)}
$$

where $f_{i}\left(u_{i}, \delta_{i}\right)=\left(1+\delta_{i}\right) u_{i}$ according to equation (7). The efficiency $\epsilon_{i}$ is usually smaller than in the case, where the traffic light is turned red as soon as a vehicle queue has been dissolved (for exceptions see Ref. [41]). Then, $\epsilon_{i}<0$ for $\delta_{i}>0$. Formula (32) also allows one to treat the case where the green time fractions $f_{i}$ and the cycle time $T_{\text {cyc }}$ are not adapted to the respective traffic situation, but where a fixed cycle time $T_{\text {cyc }}=T_{\text {cyc }}^{0}$ and fixed green time fractions $f_{i}^{0}$ are implemented. This corresponds to constant green times

$$
f_{i}^{0} T_{\text {cyc }}^{0}\left(\left\{f_{j}^{0}\right\}\right)=\frac{f_{i}^{0} T_{\mathrm{los}}}{1-\sum_{j} f_{j}^{0}}
$$

In case of uniform vehicle arrivals, we just have to insert the corresponding value $f_{i}=f_{i}^{0}$ into equation (32) to obtain $\epsilon_{i}$. In the case of non-uniform arrivals, $\epsilon_{i}$ can be understood as fit parameter of our model, which allows us to adjust our formulas to empirical data and to quantify the efficiency of traffic light operation. In this way, we can also absorb effects of stochastic vehicle arrivals into the efficiency coefficients $\epsilon_{i}$, which simplifies our treatment a lot.

\section{Fundamental relationships for undersaturated traffic}

The travel time is generally given by the sum of the free travel time $\mathcal{T}_{i}^{0}=L_{i} / V_{i}^{0}$ and the average delay time $\mathcal{T}_{i}^{\text {av }}$, where $L_{i}$ denotes the length of the road section used by vehicle stream $i$ and $V_{i}^{0}$ the free speed (or speed limit). With equation (24), we get

$\mathcal{T}_{i}\left(\left\{u_{j}\right\}, \epsilon_{i}\right)=\mathcal{T}_{i}^{0}+\mathcal{T}_{i}^{\mathrm{av}}\left(\left\{u_{j}\right\}, \epsilon_{i}\right)=\frac{L_{i}}{V_{i}^{0}}+\frac{\Delta N_{i}^{\mathrm{av}}\left(\left\{u_{j}\right\}, \epsilon_{i}\right)}{u_{i} \widehat{Q}_{i}}$.

Inserting equation (29), we can express the travel time solely in terms of the utilization $u_{i}$, and we have

$$
\mathcal{T}_{i}\left(\left\{u_{j}\right\}, \epsilon_{i}\right)=\frac{L_{i}}{V_{i}^{0}}+\left(1-\epsilon_{i}\right) \frac{\left(1-u_{i}\right) T_{\mathrm{los}}}{2\left(1-\sum_{j} u_{j}\right)} .
$$

The formula (35) constitutes a fundamental relationship between the average travel time $\mathcal{T}_{i}^{\text {av }}$ on the capacity utilization $u_{i}$ under the assumptions made (mainly cyclical operation with certain efficiencies $\epsilon_{i}$ ). Of course, one still needs to specify the factor $\left(1-\epsilon_{i}\right)$. In case of constant arrival rates $A_{i}$, this factor is given by equation (32), which finally results in

$$
\mathcal{T}_{i}\left(u_{i},\left\{f_{j}\right\}\right)=\frac{L_{i}}{V_{i}^{0}}+\frac{\left(1-f_{i}\right)^{2}}{\left(1-u_{i}\right)} \frac{T_{\text {los }}}{2\left(1-\sum_{j} f_{j}\right)} .
$$

After insertion of equation (7), we get

$$
\mathcal{T}_{i}\left(\left\{u_{j}\right\},\left\{\delta_{j}\right\}\right)=\frac{L_{i}}{V_{i}^{0}}+\frac{\left[1-\left(1+\delta_{i}\right) u_{i}\right]^{2} T_{\mathrm{los}}}{\left(1-u_{i}\right) 2\left[1-\sum_{j}\left(1+\delta_{j}\right) u_{j}\right]} .
$$

Sometimes, it is desireable to express the fundamental relationships in terms of the density rather than the utility. Inserting equation (35) into $\mathcal{T}_{i}^{\text {av }}\left(u_{i}, \epsilon_{i}\right)=\mathcal{T}_{i}\left(u_{i}, \epsilon_{i}\right)-$ $L_{i} / V_{i}^{0}$, and this into equation (26), we obtain the equation

$$
\rho_{i}^{\mathrm{av}}\left(\left\{u_{j}\right\}, \epsilon_{i}, L_{i}\right)=\frac{u_{i} \widehat{Q}_{i}}{L_{i}}\left(1-\epsilon_{i}\right) \frac{\left(1-u_{i}\right) T_{\mathrm{los}}}{2\left(1-\sum_{j} u_{j}\right)},
$$

which can be numerically inverted to give the utilization $u_{i}$ as a function of the scaled densities $\rho_{j}^{\text {av }} L_{j} /\left(1-\epsilon_{j}\right)$. The calculations are simpler in case of a fixed cycle time $T_{\text {cyc }}^{0}$ and an uniform arrival of vehicles. By inserting equation (20) 
into (26) [and with $f_{i}=f_{i}^{0}, T_{\text {cyc }}=T_{\text {cyc }}^{0}$, see Eq. (33)], we get

$$
\begin{aligned}
L_{i} \rho_{i}^{\mathrm{av}}\left(u_{i},\left\{f_{j}^{0}\right\}\right) & =\Delta N_{i}^{\mathrm{av}}\left(u_{i},\left\{f_{j}^{0}\right\}\right) \\
& =u_{i} \widehat{Q}_{i} \frac{\left(1-f_{i}^{0}\right)^{2} T_{\mathrm{cyc}}^{0}\left(\left\{f_{j}^{0}\right\}\right)}{2\left(1-u_{i}\right)},
\end{aligned}
$$

which finally yields

$$
u_{i}\left(\rho_{i}^{\mathrm{av}} L_{i},\left\{f_{j}^{0}\right\}\right)=\left(1+\left(1-f_{i}^{0}\right)^{2} \frac{\widehat{Q}_{i} T_{\mathrm{cyc}}^{0}\left(\left\{f_{j}^{0}\right\}\right)}{2 \rho_{i}^{\mathrm{av}} L_{i}}\right)^{-1} .
$$

This can be inserted into equation (26) to give

$$
\begin{aligned}
\mathcal{T}_{i}^{\mathrm{av}}\left(\rho_{i}^{\mathrm{av}} L_{i},\left\{f_{j}^{0}\right\}\right) & =\frac{\rho_{i}^{\mathrm{av}} L_{i}}{u_{i}\left(\rho_{i}^{\mathrm{av}} L_{i},\left\{f_{j}^{0}\right\}\right) \widehat{Q}_{i}} \\
& =\frac{\rho_{i}^{\mathrm{av}} L_{i}}{\widehat{Q}_{i}}+\left(1-f_{i}^{0}\right)^{2} \frac{T_{\mathrm{cyc}}^{0}\left(\left\{f_{j}^{0}\right\}\right)}{2} .
\end{aligned}
$$

\subsection{Transition to congested traffic}

The utilizations $u_{i}$ increase proportionally to the arrival flows $A_{i}$, i.e. they go up during the rush hour. Eventually,

$$
\sum_{j} f_{j}=\sum_{j}\left(1+\delta_{j}\right) u_{j} \rightarrow 1
$$

which means that the intersection capacity is reached. Sooner or later, there will be no excess capacities anymore, which implies $\delta_{i} \rightarrow 0$ and $f_{i} \rightarrow u_{i}$. In this case, we do not have any finite time periods $\Delta T_{i}-T_{i}$, during which there are no delayed vehicles and where the departure flow $O_{i}(t)$ agrees with the arrival flow $A_{i}$. Therefore, $O_{i}(t)=\gamma_{i}(t) \widehat{Q}_{i}$ according to equation (12), and certain relationships simplify. For example, the utilization is given as integral of the permeability over one cycle time $T_{\text {cyc }}$, divided by the cycle time itself:

$$
u_{i}=\frac{1}{T_{\mathrm{cyc}}} \int_{t_{i 0}}^{t_{i 0}+T_{\mathrm{cyc}}} d t^{\prime} \gamma_{i}\left(t^{\prime}\right) .
$$

Moreover, in the case of constant in- and outflows, i.e. linear increase and decrease of the queue length, the average number $\Delta N_{i}^{\text {av }}$ of delayed vehicles is just given by half of the maximum number of delayed vehicles ${ }^{1}$

$$
\Delta N_{i}^{\mathrm{av}}=\frac{\Delta N_{i}^{\max }}{2} .
$$

\footnotetext{
1 Note that the formulas derived in this paper are exact under the assumption of continuous flows. The fact that vehicle flows consist of discrete vehicles implies deviations from our formulas of upto 1 vehicle, which slightly affects the average travel times as well. In Figure 2, for example, the number of vehicles in the queue is $N_{i}^{\max }-1$ rather than $N_{i}^{\max }$. Therefore, the related maximum delay time is $\left(1-u_{i}\right) T_{\text {cyc }}\left(N_{i}^{\max }-1\right) / N_{i}^{\max }$, which reduces the average delay time $\mathcal{T}_{i}^{\text {av }}$ by $\left(1-u_{i}\right) T_{\text {cyc }} /\left(2 N_{i}^{\max }\right)$.
}

As a consequence, we have

$$
\mathcal{T}_{i}\left(\left\{u_{j}\right\}\right)=\frac{L_{i}}{V_{i}^{0}}+\frac{\left(1-u_{i}\right) T_{\mathrm{los}}}{2\left(1-\sum_{j} u_{j}\right)} .
$$

The average speed $V_{i}^{\text {av }}$ of traffic stream $i$ is often determined by dividing the length $L_{i}$ of the road section reserved for it by the average travel time $\mathcal{T}_{i}=\mathcal{T}_{i}{ }^{0}+\mathcal{T}_{i}{ }^{\text {av }}$, which gives

$$
V_{i}^{\mathrm{av}}\left(\left\{u_{j}\right\}\right)=\frac{L_{i}}{\mathcal{T}_{i}\left(\left\{u_{j}\right\}\right)}=\left(\frac{1}{V_{i}^{0}}+\frac{\left(1-u_{i}\right) T_{\mathrm{los}}}{2 L_{i}\left(1-\sum_{j} u_{j}\right)}\right)^{-1}
$$

and can be generalized with equation (28) to cases with an efficiencies $\epsilon_{i} \neq 0$ :

$$
V_{i}^{\mathrm{av}}\left(\left\{u_{j}\right\}, \epsilon_{i}\right)=\left(\frac{1}{V_{i}^{0}}+\left(1-\epsilon_{i}\right) \frac{\left(1-u_{i}\right) T_{\mathrm{los}}}{2 L_{i}\left(1-\sum_{j} u_{j}\right)}\right)^{-1} .
$$

Note, however, that the above formulas for the average speed are implicitly based on a harmonic rather than an arithmetic average. When correcting for this, equation (46), for example, becomes

$$
V_{i}^{\mathrm{av}}\left(\left\{u_{j}\right\}\right)=\frac{L_{i} u_{i} \widehat{Q}_{i}}{\Delta N_{i}^{\max }\left(\left\{u_{j}\right\}\right)} \ln \left|1+\frac{\Delta N_{i}^{\max }\left(\left\{u_{j}\right\}\right)}{u_{i} \widehat{Q}_{i} \mathcal{T}_{i}^{0}}\right| .
$$

As is shown in Appendix A, this has a similar Taylor approximation as the harmonic average (47). The latter is therefore reasonable to use, and it is simpler to calculate.

As expected from queuing theory, the average travel time (45) diverges, when the sum of utilizations reaches the intersection capacity, i.e. $\sum_{j} u_{j} \rightarrow 1$. In this practically relevant case, the traffic light would not switch anymore, which would frustrate drivers. For this reason, the cycle time is limited to a finite value

$$
T_{\text {cyc }}^{\max }\left(\left\{u_{j}^{0}\right\}\right)=\frac{T_{\text {los }}}{1-\sum_{j} u_{j}^{0}},
$$

where typically $u_{j}^{0} \leq u_{j}$. This implies that the sum of utilizations must fulfill

$$
\sum_{j} u_{j} \leq \sum_{j} u_{j}^{0}=1-\frac{T_{\mathrm{los}}}{T_{\mathrm{cyc}}^{\max }}
$$

As soon as this condition is violated, we will have an increase of the average number of delayed vehicles in time, which characterizes the congested regime discussed in the next section.

\section{Fundamental relationships for congested traffic conditions}

In the congested regime, the number of delayed vehicles does not reach zero anymore, and platoons cannot be served without delay. Vehicles will usually have to 
wait several cycle times until they can finally pass the traffic light. This increases the average delay time enormously. It also implies that there are no excess green times, which means $\delta_{i}=0$. Consequently, we can also assume $O_{i}(t)=\widehat{Q}_{i}$, as long as the outflow from road sections during the green phase is not (yet) obstructed (otherwise see Sect. 5). Formula (13) applies again and implies for time-independent arrival flows $A_{i}$

$$
\begin{aligned}
\Delta N_{i}\left(t_{i 0}+k T_{\mathrm{cyc}}^{\max }\right) & =\Delta N_{i}\left(t_{i 0}\right)+\int_{t_{i 0}}^{t_{i 0}+k T_{\text {cyc }}^{\max }} d t^{\prime}\left[A_{i}-\gamma_{i}\left(t^{\prime}\right) \widehat{Q}_{i}\right] \\
& =\Delta N_{i}\left(t_{i 0}\right)+\left(A_{i}-u_{i}^{0} \widehat{Q}_{i}\right) k T_{\mathrm{cyc}}^{\max }, \quad(51)
\end{aligned}
$$

where

$$
u_{i}^{0}=\frac{1}{k T_{\text {cyc }}^{\max }} \int_{t_{i 0}}^{t_{i 0}+k T_{\text {cyc }}^{\max }} d t^{\prime} \gamma_{i}\left(t^{\prime}\right)<u_{i}=\frac{A_{i}}{\widehat{Q}_{i}}
$$

is the green time fraction of the cycle time $T_{\text {cyc }}^{\max }$ reserved for vehicle stream $i$. Therefore, the number of delayed vehicles grows by an amount $\left(A_{i}-u_{i}^{0} \widehat{Q}_{i}\right) T_{\text {cyc }}^{\max }=$ $\left(u_{i}-u_{i}^{0}\right) \widehat{Q}_{i} k T_{\text {cyc }}^{\max }$ in each cycle time $T_{\text {cyc }}^{\max }$. The minimum number during one cycle is

$$
\Delta N_{i}^{\min }\left(u_{i}, k\right)=\Delta N_{i}\left(t_{i 0}\right)+\left(u_{i}-u_{i}^{0}\right) \widehat{Q}_{i} k T_{\text {cyc }}^{\max },
$$

and considering equation (15), the maximum number of delayed vehicles is

$$
\Delta N_{i}^{\max }\left(u_{i}, k\right)=\Delta N_{i}^{\min }\left(u_{i}, k\right)+u_{i}\left(1-u_{i}^{0}\right) \widehat{Q}_{i} T_{\mathrm{cyc}}^{\max } .
$$

Because of $\Delta N_{i}^{\mathrm{av}}=\left(\Delta N_{i}^{\min }+\Delta N_{i}^{\max }\right) / 2$ and $A_{i}=u_{i} \widehat{Q}_{i}$, the average number of delayed vehicles is

$$
\begin{aligned}
\Delta N_{i}^{\mathrm{av}}\left(u_{i},\left\{u_{j}^{0}\right\}, k\right)= & \left(\left(u_{i}-u_{i}^{0}\right) k\right. \\
& \left.+\frac{u_{i}\left(1-u_{i}^{0}\right)}{2}\right) \widehat{Q}_{i} T_{\mathrm{cyc}}^{\max }\left(\left\{u_{j}^{0}\right\}\right) .
\end{aligned}
$$

Obviously, the average density

$$
\begin{aligned}
\rho_{i}^{\mathrm{av}}\left(u_{i},\left\{u_{j}^{0}\right\}, L_{i}, k\right)= & \left(\left(u_{i}-u_{i}^{0}\right) k\right. \\
& \left.+\frac{u_{i}\left(1-u_{i}^{0}\right)}{2}\right) \frac{\widehat{Q}_{i} T_{\mathrm{cyc}}^{\max }\left(\left\{u_{j}^{0}\right\}\right)}{L_{i}}
\end{aligned}
$$

is obtained by dividing the previous formula by $L_{i}$.

It seems logical to use equation (55) to determine the average delay time as

$$
\begin{aligned}
\mathcal{T}_{i}^{\mathrm{av}}\left(u_{i},\left\{u_{j}^{0}\right\}, k\right)= & \frac{\Delta N_{i}^{\mathrm{av}}\left(u_{i},\left\{u_{j}^{0}\right\}, k\right)}{A_{i}} \\
= & \left(1-\frac{u_{i}^{0}}{u_{i}}\right) k T_{\mathrm{cyc}}^{\max }\left(\left\{u_{j}^{0}\right\}\right) \\
& +\frac{\left(1-u_{i}^{0}\right) T_{\mathrm{cyc}}^{\max }\left(\left\{u_{j}^{0}\right\}\right)}{2} .
\end{aligned}
$$

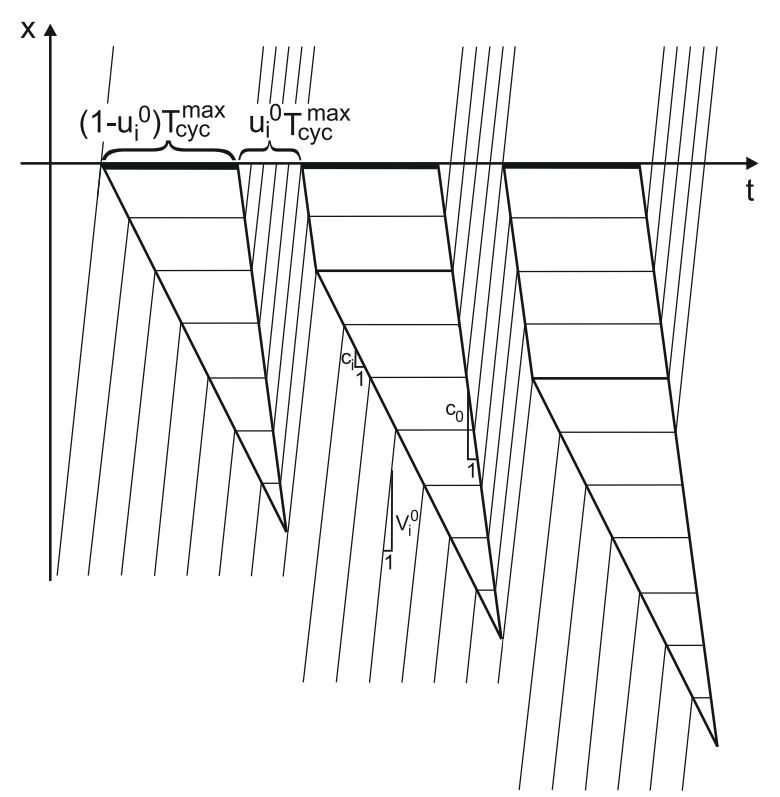

Fig. 3. Schematic illustration of growing vehicle queues in the congested regime. Since not all vehicles can be served within one green time $u_{i}^{0} T_{\text {cyc }}^{\max }$, vehicles are forced to make additional stops, which correspond to the rhomboidal parts of the queue in the above time-space diagram. The triangular part, in contrast, represents newly arriving vehicles.

However, this turns out to be not correct, as some of this time is actually not lost, but used to move forward (see Fig. 3). After this first simple analysis, we need to look at the problem more carefully, and perform the calculation without the use of equation (24), as Little's Law is not applicable in case of non-stable, systematically growing queues.

Again, we apply the fact that the number of vehicles arriving in one cycle is $A_{i} T_{\text {cyc }}^{\max }$, while the number of vehicles served during one green time period of duration $u_{i}^{0} T_{\text {cyc }}^{\max }$ is given by $\widehat{Q}_{i} u_{i}^{0} T_{\text {cyc }}^{\max }$. The difference of both numbers is added to the growing vehicle queue. The quotient of the number $k A_{i} T_{\text {cyc }}^{\max }$ of vehicles arriving in $k$ cycles and the number $\widehat{Q}_{i} u_{i}^{0} T_{\text {cyc }}^{\max }$ of vehicles served during one green time period, when rounded down, corresponds to the number $n_{\mathrm{s}}$ of additional stops needed by newly arriving vehicles. Therefore, with $A_{i}=u_{i} \widehat{Q}_{i}$ we have

$$
n_{\mathrm{s}}\left(u_{i},\left(\left\{u_{j}^{0}\right\}\right), k\right)=\left\lfloor\frac{A_{i} k T_{\mathrm{cyc}}^{\max }\left(\left\{u_{j}^{0}\right\}\right)}{\widehat{Q}_{i} u_{i}^{0} T_{\mathrm{cyc}}^{\max }\left(\left\{u_{j}^{0}\right\}\right)}\right\rfloor=\left\lfloor\frac{u_{i} k}{u_{i}^{0}}\right\rfloor .
$$

Here, $\lfloor x\rfloor$ represents the floor function rounding to lower integers (i.e. cutting the digits after the period, if $x>0$ ). Assuming that $t_{i 0}$ is the time at which congestion sets in, equation (58) can be generalized to continuous time $t$, allowing us to estimate the number of additional stops of a vehicle arriving at time $t$ :

$n_{\mathrm{s}}\left(u_{i},\left\{u_{j}^{0}\right\}, t\right)=\left\lfloor\frac{A_{i}\left(t-t_{i 0}\right)}{\widehat{Q}_{i} u_{i}^{0} T_{\mathrm{cyc}}^{\max }\left(\left\{u_{j}^{0}\right\}\right)}\right\rfloor=\left\lfloor\frac{u_{i}\left(t-t_{i 0}\right)}{u_{i}^{0} T_{\mathrm{cyc}}^{\max }\left(\left\{u_{j}^{0}\right\}\right)}\right\rfloor$. 
As Figure 3 shows, the delay time by each of the $n_{\mathrm{s}}$ additional stops is $\left(1-u_{i}^{0}\right) T_{\text {cyc }}^{\max }$. Moreover, we can see that the triangular part of the vehicle queue in the space-time plot gives a further contribution to the delay time of vehicles. Applying equation (20) with $f_{i}=u_{i}=u_{i}^{0}$, the average time delay in this triangular part is $\left(1-u_{i}^{0}\right) T_{\text {cyc }}^{\max } / 2$, i.e. the arithmetic average between zero and the sum of the red and amber time period (amounting to $\left(1-u_{i}^{0}\right) T_{\text {cyc }}^{\max }$ ). In summary, the delay time of a newly arriving vehicle at time $t$ (when averaging over the triangular part for the sake of simplicity), is

$$
\begin{aligned}
\mathcal{T}_{i}^{\mathrm{av}}\left(u_{i}, t\right) & =\left(1-u_{i}^{0}\right) \frac{T_{\mathrm{cyc}}^{\max }}{2}+\left\lfloor\frac{u_{i}\left(t-t_{i 0}\right)}{u_{i}^{0} T_{\mathrm{cyc}}^{\max }}\right\rfloor\left(1-u_{i}^{0}\right) T_{\mathrm{cyc}}^{\max } \\
& =\left(\frac{1}{2}+\left\lfloor\frac{u_{i}\left(t-t_{i 0}\right)}{u_{i}^{0} T_{\mathrm{cyc}}^{\max }}\right\rfloor\right)\left(1-u_{i}^{0}\right) T_{\mathrm{cyc}}^{\max }
\end{aligned}
$$

Accordingly, the average travel time does not only grow with time $t$ (or the number $k$ of cycles passed), it also grows stepwise due to the floor function $\lfloor x\rfloor$.

When we also average formula (60) over its steps, we obtain the approximate relationship

$$
\begin{aligned}
\mathcal{T}_{i}^{\mathrm{av}}\left(u_{i}, t\right) & \approx\left(\frac{u_{i}\left(t-t_{i 0}\right)}{u_{i}^{0} T_{\mathrm{cyc}}^{\max }}\right)\left(1-u_{i}^{0}\right) T_{\mathrm{cyc}}^{\max } \\
& =u_{i}\left(t-t_{i 0}\right) \frac{\left(1-u_{i}^{0}\right)}{u_{i}^{0}}
\end{aligned}
$$

where it is important to consider that the floor function $\lfloor x\rfloor$ is shifted by 0.5 with respect to the function $\operatorname{round}(x)$, which rounds to the closest integer: $\operatorname{round}(x)=\lfloor x+0.5\rfloor$. Moreover, when averaging over the last $(k+1$ st $)$ cycle we get

$$
\mathcal{T}_{i}^{\mathrm{av}}\left(u_{i}, k\right) \approx u_{i}\left(k+\frac{1}{2}\right) \frac{1-u_{i}^{0}}{u_{i}^{0}} T_{\text {cyc }}^{\max } .
$$

It is also possible to replace the dependence on the number $k$ of cycles by a dependence on the average density of delayed vehicles by applying equation (55). In this way, we obtain

$$
k+\frac{1}{2}=\frac{\Delta N_{i}^{\mathrm{av}}\left(u_{i},\left\{u_{j}^{0}\right\}, k\right)}{\left(u_{i}-u_{i}^{0}\right) \widehat{Q}_{i} T_{\mathrm{cyc}}^{\max }\left(\left\{u_{j}^{0}\right\}\right)}-\frac{u_{i}^{0}\left(1-u_{i}\right)}{2\left(u_{i}-u_{i}^{0}\right)} .
$$

Therefore, while in Section 3 we could express the average travel time and the average velocity either in dependence of the average density $\rho_{i}^{\text {av }}$ or the utilization $u_{i}$ alone, we now have a dependence on both quantities.

Finally note that equations (60) to (62) may be generalized to the case where the arrival rate of vehicles is not time-independent. This changes the triangular part of Figure 3. In order to reflect this, the corresponding contribution $\left(1-u_{i}^{0}\right) T_{\text {cyc }}^{\max } / 2$ may, again, be multiplied with a prefactor $\left(1-\epsilon_{i}\right)$, which defines an efficiency $\epsilon_{i}$. While $\epsilon_{i}=0$ corresponds to the previously discussed case of an uniform arrival of vehicles, $\epsilon_{i}=1$ reflects the case where a densely packed platoon of vehicles arrives at the moment when the last vehicle in the queue has started to move forward.

\section{Fundamental relationships for oversaturated traffic conditions}

We have seen that, under congested conditions, the number of delayed vehicles is growing on average. Hence, the vehicle queue will eventually fill the road section reserved for vehicle stream $i$ completely. Its maximum storage capacity per lane for delayed vehicles is

$$
\Delta N_{i}^{\mathrm{jam}}\left(L_{i}\right)=L_{i} \rho_{i}^{\mathrm{jam}}
$$

where $\rho_{i}^{\mathrm{jam}}$ denotes the maximum density of vehicles per lane. The road section becomes completely congested at the time

$$
t=t_{i 0}+k T_{\mathrm{cyc}}^{\max }+\Delta t
$$

when $\Delta N_{i}^{\min }\left(u_{i}, k\right)+A_{i} \Delta t$ according to equation (53) reaches the value $\Delta N_{i}^{\mathrm{jam}}$, which implies

$$
\Delta t=\frac{\Delta N_{i}^{\mathrm{jam}}-\Delta N_{i}^{\mathrm{min}}\left(u_{i}, k\right)}{A_{i}},
$$

where

$$
k=\left\lfloor\frac{\Delta N_{i}^{\mathrm{jam}}}{\left(u_{i}-u_{i}^{0}\right) T_{\mathrm{cyc}}^{\max }}\right\rfloor,
$$

see equation (53). The number $n_{\mathrm{s}}+1$ of stops is given by equation (59). See Figure 4 for an illustration.

Complete congestion causes spillover effects and obstructs the arrival of upstream vehicles, even though the green phase would, in principle, allow them to depart. When these spill-over effects set in, we are in the oversaturated regime, and only a certain fraction $\sigma_{i}$ of the green phase of duration $u_{i}^{0} T_{\text {cyc }}^{\max }$ can be used, where $0 \leq$ $\sigma_{i} \leq 1$. Note that $\sigma_{i}$ decreases in time as the number of blocked subsequent road sections grows. This may eventually lead to gridlock in a large area of the urban traffic system.

In the case $\sigma_{i}<1, f_{i}=u_{i}^{0}$ must essentially be replaced by $f_{i}=\sigma_{i} u_{i}^{0}$ in the fundamental relationships for congested traffic, and the lost service time becomes

$$
T_{\mathrm{los}}=\sum_{i}\left[\tau_{i}+\left(1-\sigma_{i}\right) u_{i}^{0} T_{\mathrm{cyc}}^{\max }\right] .
$$

That is, the reduced service time may be imagined like an extension of the amber time periods. Therefore, it would make sense to reduce the cycle time to a value $T_{\text {cyc }}<T_{\text {cyc }}^{\max }$ in the oversaturated regime.

Note, however, that the travel times on the road section reserved for stream $i$ are not growing anymore, because the road section is limited to a length $L_{i}$. This allows us to determine the corresponding travel time on the link as follows: the number of vehicles served per cycle time is $\sigma_{i} u_{i}^{0} T_{\text {cyc }}^{\max } \widehat{Q}_{i}$. For this reason, the average travel time can be estimated as

$$
\mathcal{T}_{i}\left(u_{i}, \sigma_{i}, L_{i}\right)=\frac{\Delta N_{i}^{\mathrm{jam}}\left(L_{i}\right)}{\sigma_{i} u_{i}^{0} T_{\mathrm{cyc}}^{\max }} T_{\mathrm{cyc}}^{\mathrm{max}}=\frac{L_{i} \rho_{i}^{\mathrm{jam}}}{\sigma_{i} u_{i}^{0} \widehat{Q}_{i}},
$$




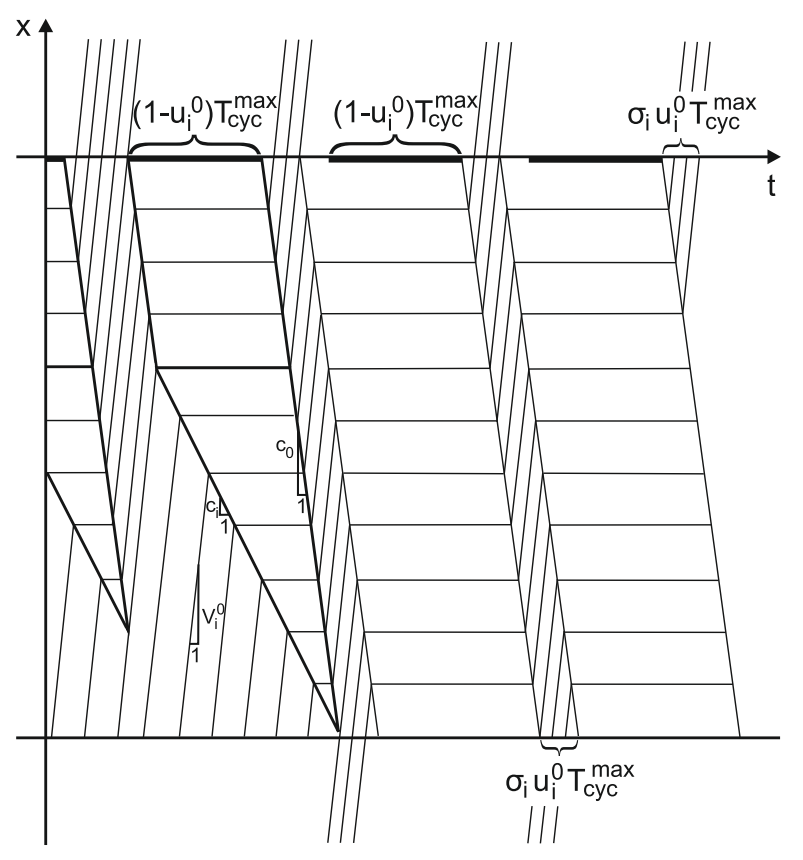

Fig. 4. Schematic illustration of the service of vehicle queues, when the road section is fully congested. The lower horizontal line indicates the location of the upstream end of the road section. It can be seen that vehicles are stopped several times, and that new vehicles can only enter when some vehicles have been served by the traffic light located at the $t$-axis, and the space freed up by this has reached the end of the vehicle queue. For similar considerations see reference [35]. Note that the characteristic speed $c_{0}$ of the shock fronts (which corresponds to the slope of the congested flow-density relationship for a road section without the consideration of traffic lights) is different from the slope of the urban fundamental diagram, because of the effect of signal offsets and delays [31]. Therefore, $c_{0}$ should be understood as fit parameter, here.

and the average delay time $\mathcal{T}_{i}^{\text {av }}=\mathcal{T}_{i}-\mathcal{T}_{i}^{0}$ as

$$
\mathcal{T}_{i}^{\text {av }}\left(u_{i}, \sigma_{i}, L_{i}\right)=\frac{L_{i} \rho_{i}^{\mathrm{jam}}}{\sigma_{i} u_{i}^{0} \widehat{Q}_{i}}-\frac{L_{i}}{V_{i}^{0}} .
$$

Note that these values are now independent of both, the utilization and the average density, as soon as the latter assumes the value $\rho_{i}^{\text {av }}=\rho_{i}^{\mathrm{jam}}$, corresponding to a fully congested road section.

\subsection{Transition from oversaturated to undersaturated traffic conditions}

If the arrival flow $A_{i}$ after the rush hour drops below the value of $\sigma_{i} u_{i}^{0} T_{\mathrm{cyc}}^{\max }$, the vehicle queue will eventually shrink, and the road section used by vehicle stream $i$ enters from the oversaturated into the congested regime. The formulas for the evolution of the number of delayed vehicles are analogous to equations (53) and (54). The queue length starts with $\Delta N_{i}^{\mathrm{jam}}$ and is reduced by $\left(u_{i}-\sigma_{i} u_{i}^{0}\right) \widehat{Q}_{i} k T_{\text {cyc }}<0$ in each cycle of length $T_{\text {cyc }}$. Counting the number of cycles since the re-entering into the congested regime by $k^{\prime}$, we have

$$
\Delta N_{i}^{\mathrm{min}}\left(u_{i}, k^{\prime}\right)=\Delta N_{i}^{\mathrm{jam}}+\left(u_{i}-\sigma_{i} u_{i}^{0}\right) \widehat{Q}_{i} k^{\prime} T_{\mathrm{cyc}},
$$

and considering equation (15), the maximum number of delayed vehicles is

$$
\Delta N_{i}^{\max }\left(u_{i}, k^{\prime}\right)=\Delta N_{i}^{\min }\left(u_{i}, k^{\prime}\right)+u_{i}\left(1-\sigma_{i} u_{i}^{0}\right) \widehat{Q}_{i} T_{\text {cyc }} .
$$

As soon as $\Delta N_{i}^{\min }\left(u_{i}, k^{\prime}\right)$ reaches zero, the road section used by vehicle stream $i$ enters the undersaturated regime. Before, the number of stops of vehicles joining the end of the vehicle queue are expected to experience an number $n_{\mathrm{s}}+1$ of stops with

$$
n_{\mathrm{s}}\left(k^{\prime}\right)=\left\lfloor\frac{u_{i} k^{\prime}}{\sigma_{i} u_{i}^{0}}\right\rfloor,
$$

compare equation (58).

\section{Summary and outlook}

Based on a few elementary assumptions, we were able to derive fundamental relationships for the average travel time $\mathcal{T}_{i}^{\text {av }}$ and average velocity $V_{i}^{\text {av }}$. These relationships are functions of the utilization $u_{i}$ of the service capacity of a cyclically signalized intersection and/or the average number $\Delta N_{i}^{\text {av }}$ of delayed vehicles (or the average density $\rho_{i}^{\text {av }}$ of vehicles in the road section of length $L_{i}$ reserved for traffic stream $i$ ). We found different formulas, (1) for the undersaturated regime, (2) for the congested regime, and (3) for the oversaturated regime. While we also discussed situations, where fixed cycle times $T_{\text {cyc }}^{0}$ are applied, we primarily focussed on situations, where the cycle time is adjusted to the utilization $u_{i}$ (in the undersaturated regime) and to the effectively usable green time fraction $\sigma_{i}$ (in the oversaturated regime). Our results are summarized in Figure 5 , where also a comparison with the capacity restraint function (1) is made.

The formulas for the non-congested regime can be either expressed as non-trivial functions of the utilization $u_{i}$ or the average queue length $\Delta N_{i}^{\text {av }}$ (or the average density $\left.\rho_{i}^{\max }\right)$. They contain a fit parameter $\epsilon_{i}$, which reflects effects of variations in the arrival flow and relates to the efficiency of traffic signal operation in terms of synchronizing with vehicle platoons. In the best case, delay times are zero, which shows the great optimization potential for traffic control in this regime.

In the congested regime, the number of delayed vehicles grows in time, and the majority of vehicles is stopped several times by the same traffic light. Therefore, the average travel time does not only depend on the utilization $u_{i}$, but also on the average vehicle queue $\Delta N_{i}^{\text {av }}$ (or the average density $\left.\rho_{i}^{\text {av }}\right)$. Although the traffic light control can still improve the average travel times by synchronizing with the arrival of vehicles, the related efficiency effect is rather limited. 


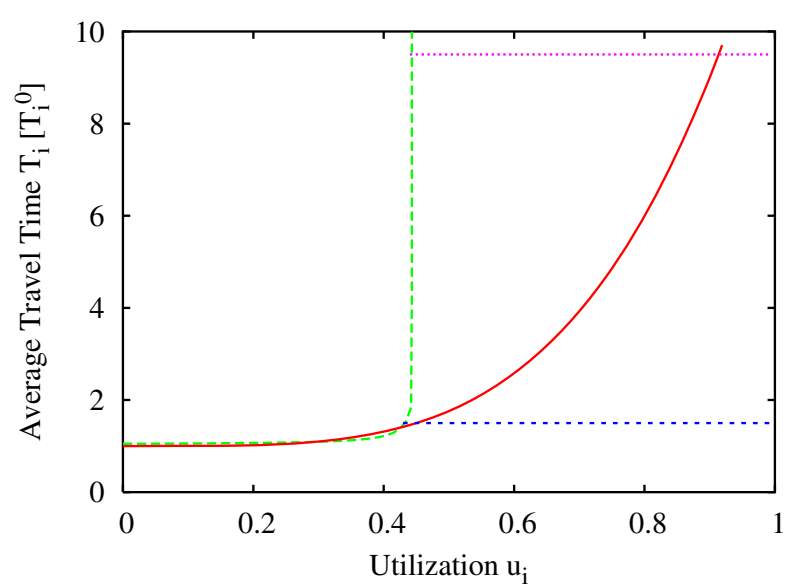

Fig. 5. (Color Online) Schematic illustration of the capacity restraint function (1) that the Bureau of Public Roads recommends to use [16] (solid line), together with analytical results of this paper (dashed lines). Travel time $\mathcal{T}_{i}$ is measured in units of $\mathcal{T}_{i}^{0}$. The green long-dashed line shows that the travel time diverges at $u_{i} \approx 0.45$, if two green phases during one cycle and identical utilizations $u_{1}=u_{2}$ of both associated road sections are assumed, furthermore, if the parameters are set to $T_{\text {los }}=0.1 \mathcal{T}_{i}^{0}$ and $\delta=0.1$. (A generalization to traffic operation with more than two green phases is easily possible.) When the cycle time is limited to a finite value $\mathcal{T}_{\text {cyc }}^{\max }$ to avoid infinite delay times in one of the vehicle queues, one will have growing vehicle queues and increasing travel times in both road sections. Therefore, the travel time can assume any value above the lower dashed horizontal line. The link travel time is only limited by the above dashed horizontal line, which corresponds to the situation where the vehicle queue fills the road section completely. Note that the capacity restraint function (solid line) averages over all travel time measurements for a given utilization $u_{i}$. In the right part of the diagram this concerns measurements that depend on the duration of congestion and scatter between both horizontal dashed lines. Here, the curve is shown for $\alpha_{i}=0.5, \beta_{i}=4$, and $A_{i} / C_{i}=u_{i} / 0.45$.

In the oversaturated regime, the storage capacity of the road section is fully occupied by delayed vehicles, which obstructs the arrival flow. Therefore, the average travel time of a road section reaches a constant maximum value. Nevertheless, the travel time of vehicles increases further in time due to spillover effects, which trigger the spreading of congestion to upstream road sections. The actually usable fraction of the green time period is described by a parameter $\sigma_{i}$. Synchronization can still reach some improvements. The most favorable control is oriented at a fluent upstream propagation of the little remaining free space (the difference between $\Delta N_{i}^{\max }$ and $\Delta N_{i}^{\min }$ ). That is, rather than minimizing the delay of downstream moving vehicle platoons, one should now minimize the delay in filling upstream moving gaps [42]. Furthermore, note that the free travel time $\mathcal{T}_{i}^{0}=L_{i} / V_{i}^{0}$ and the delay time in the oversaturated regime are proportional to the length $L_{i}$ of the road section, while the delay times in the undersaturated and congested regimes are independent of $L_{i}$.
Based on the above results, it is obvious that it cannot be very successful to describe the travel time of a link by a capacity restraint function which depends on $A_{i} / C_{i}=$ $u_{i} / u_{i}^{0}$ only. This basically means an averaging over data that, in principle, are also dependent on the average queue length $\Delta N_{i}^{\text {av }}$ (or on the time passed since the onset of congestion). It is, therefore, no wonder that empirical data of travel times as a function of the utilization scatter so enormously in the congested and oversaturated regimes (see e.g. Ref. [27]), that a fitting of the data to functional dependencies of any kind does not make much sense.

This has serious implications for transport modeling, as capacity restraint functions such as formula (1) are used for modeling route choice and, hence, for traffic assignment. Based on the necessary revision of this formula and comparable ones, all traffic scenarios based on these formulas should be critically questioned. Given the computer power of today, it would not constitute a problem to perform a dynamic traffic assignment and routing based on the more differentiated formulas presented in this paper.

\subsection{Transferring the link-based urban fundamental diagrams to an area-based one}

We may finally ask ourselves, whether the above formulas would also allow one to make predictions about the average travel times and speeds for a whole area of an urban traffic network, rather than for single road sections ("links") only. This would correspond to averaging over the link-based fundamental diagrams of that area. For the sake of simplicity, let us assume for a moment that the parameters of all links would be the same, and derive a velocity-density diagram from the relationship (A.9) between average vehicle speed $V_{i}^{\text {av }}$ and the capacity utilization $u_{i}$. Taking into account $V_{i}^{0}=L_{i} / \mathcal{T}_{i}^{0}$ and dropping the index $i$, we can write

$$
V^{\mathrm{av}}(u)=\frac{V^{0} \ln \left\{1+[1-f(u)] T_{\mathrm{cyc}} / \mathcal{T}^{0}\right\}}{(1-u) T_{\mathrm{cyc}} / \mathcal{T}^{0}}+V^{0} \frac{f(u)-u}{1-u},
$$

with $f(u)=(1+\delta) u$. The cycle time

$$
T_{\mathrm{cyc}}=\frac{T_{\mathrm{los}}}{1-s f(u)}
$$

follows from equation (5), assuming $s$ signal phases with $f_{i}=f$ for simplicity. Note that the previous formulas for $\rho_{i}^{\text {av }}$ denote the average density of delayed vehicles, while the average density of all vehicles (i.e. delayed and freely moving ones) is given by

$$
\rho(u)=\frac{N(u)}{L}=\frac{N(u) / \mathcal{T}(u)}{L / \mathcal{T}(u)}=\frac{A(u)}{V^{\mathrm{av}}(u)}=\frac{u \widehat{Q}}{V^{\mathrm{av}}(u)},
$$

where $N=A \mathcal{T}=u \widehat{Q} \mathcal{T}$ denotes the average number of vehicles on a road section of length $L$. Plotting $V^{\text {av }}(u)$ over $\rho(u)$ finally gives a speed-density relationship $V^{\text {av }}(\rho)$.

We have now to address the question of what happens, if we average over the different road sections of an urban 
area. Considering the heterogeneity of the link lengths $L_{i}$, efficiencies $\epsilon_{i}$, utilizations $u_{i}$, and densities $\rho_{i}^{\text {av }}$, one could think that the spread in the data would be enormous. However, a considerable amount of smoothing results from the fact that in- and outflows of links within the studied urban area cancel out each other, and it does not matter whether a vehicle is delayed in a particular link, or in the previous or subsequent one. Therefore, the resulting fundamental diagrams for urban areas are surprisingly smooth $[29,30]$. The details of the curves, however, are expected to depend not only on the average density, but also on the density distribution, the signal operation schemes, and potentially other factors as well.

When averaging over different links, we have to study the effects of the averaging procedure on the density and the speed. The density just averages linearly: thanks to Little's law [39], the formula (76) can also be applied to an urban area, as long as the average number of vehicles in it is stationary. However, as the link-based fundamental diagram between the flow $Q(\rho)=\rho V^{\text {av }}(\rho)$ and the density $\rho$ is convex, evaluating the flow at some average density overestimates the average flow $^{2}$. This also implies that, when the relationship $V^{\text {av }}(\rho)$ is transferred from single links to urban areas, the average speed is overestimated for a given density, as reference [31] has shown.

Despite this expected deviation in heterogeneous and inhomogeneously used road networks, and despite the many other simplifications, the curve $V^{\text {av }}(\rho)$ fits empirical data of the speed-density relation in an urban area quite well. Figure 6 displays empirical data obtained for the center of Yokohama [29] together with a fit of the theoretical speed-density relationship $V^{\text {av }}(\rho)$, where only the three parameters $V_{0}, \delta$, and $V^{*}=L / T_{\text {los }}$ were adjusted. Surprisingly, the effects of network interactions could be sufficiently well represented by a single parameter $\delta$, which relates to the efficiency $\epsilon$ of road sections according to equation (32). This approximation seems to work in situations close enough to a statistical equilibrium (when the number of vehicles in the urban area does not change too quickly).

In contrast, for an understanding of the spreading dynamics of congestion patterns, we expect that one must study the interaction between the flow dynamics and the network structure. This difficult subject goes beyond the scope of this paper and beyond what is doable at the moment, but it will be interesting to address it in the future.

\footnotetext{
${ }^{2}$ When averaging over speed values, they have to be weighted by the number of vehicles concerned, i.e. by the density. This comes down to determining an arithmetic average of the flow values and dividing the result by the arithmetic average of the related densities. Hence, an overestimation of the average flow also implies an overestimation of the average velocity. However, considering the curvature of $Q(\rho)$ and knowing the variability of the density $\rho$ allows one to estimate correction terms.

3 This corresponds to $10 \%$ extra green time, an average distance between successive traffic lights of roughly $100 \mathrm{~m}$ (depending on $T_{\text {los }}$ ), and an average of 3 traffic phases (which appears plausible, considering that there are many one-way roads,
}

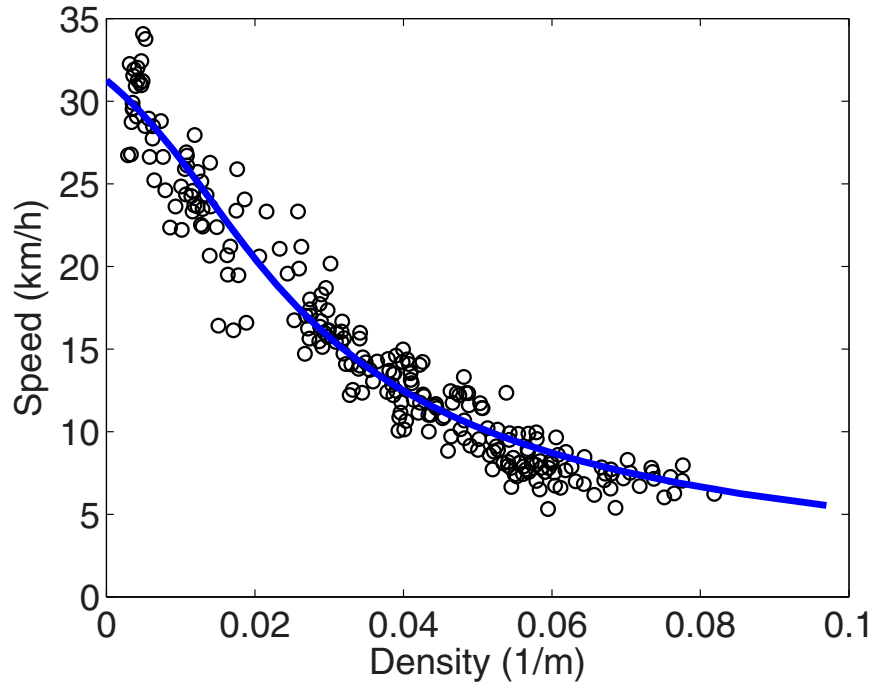

Fig. 6. (Color online) Fundamental velocity-density relationship for a central area of Yokohama. Small circles correspond to empirical data by Kuwahara as evaluated by Daganzo and Geroliminis [31]. The fit curve corresponds to the theoretically derived equations (74) to (76), where the outflow (discharge flow) $\widehat{Q}=1800$ veh./h/lane and the free speed $V^{0}=50 \mathrm{~km} / \mathrm{h}$ have been fixed. The only fit parameters were $\delta=0.1, T_{\mathrm{los}} / \mathcal{T}_{0}=1.4$, and $s=3^{3}$.

The author thanks for an inspiring presentation by Carlos Daganzo, for useful comments by Stefan Lämmer, and intresting discussions with Nikolas Geroliminis, who was also kind enough to provide the empirical data from the center of Yokohama displayed in Figure 6, see Figure 7 in reference [29]. He extracted these from original data of GPS-equipped taxis by Prof. Masao Kuwahara from the University of Tokyo. The fit of the theoretically predicted relationship to the empirical data was carried out by Anders Johansson. Furthermore, the author is grateful for partial support by the DaimlerBenz Foundation Project 25-01.1/07 on BioLogistics, the VW Foundation Project I/82 697, the NAP project KCKHA005 "Complex Self-Organizing Networks of Interacting Machines: Principles of Design, Control, and Functional Optimization", and the ETH Competence Center 'Coping with Crises in Complex Socio-Economic Systems' (CCSS) through ETH Research Grant CH1-01-08-2.

\section{Appendix A: Determination of average travel times and velocities}

Let $f(x)$ be a function and $w(x)$ a weight function. Then, the average of the function between $x=x_{0}$ and $x=x_{1}$ is

which need less than 4 phases in one cycle time). Consequently, all parameters are quite reasonable (see also Ref. [31]). Note that effects of oversaturation did not have to be considered in Figure 6. This is, in fact, consistent with pictures from Google Earth. 
defined as

$$
\frac{\int_{x_{0}}^{x_{1}} d x^{\prime} w\left(x^{\prime}\right) f\left(x^{\prime}\right)}{\int_{x_{0}}^{x_{1}} d x^{\prime} w\left(x^{\prime}\right)} .
$$

In case of uniform arrivals of vehicles, we have a functional relationship of the form $f(x)=a+b x$ for the travel time, and the weigth function is constant, i.e. $w(x)=w$. Here, $a=\mathcal{T}_{i}^{0}, x=\Delta N_{i}$, and $b=1 / A_{i}=1 /\left(u_{i} \widehat{Q}_{i}\right)$. With $x_{0}=0$ and $x_{1}=\Delta N_{i}^{\max }$, the formula for the average travel time becomes

$$
\frac{w\left[\left(a x_{1}+b x_{1}^{2} / 2\right)-\left(a x_{0}+b x_{0}^{2} / 2\right)\right]}{w\left(x_{1}-x_{0}\right)}=a+b \frac{x_{1}+x_{0}}{2},
$$

where we have used $\left(x_{1}^{2}-x_{0}^{2}\right)=\left(x_{1}-x_{0}\right)\left(x_{1}+x_{0}\right)$. Inserting the above parameters, we obtain the previously derived result

$$
\mathcal{T}_{i}=\mathcal{T}_{i}^{0}+\frac{\Delta N_{i}^{\max }}{2 u_{i} \widehat{Q}_{i}}
$$

When determining the average velocity $V_{i}^{\text {av }}$, the function to average over is of the form $f(x)=c /(a+b x)$, where $c=L_{i}$ and the other parameters are as defined before. We use the relationship

$$
\begin{aligned}
\int_{x_{0}}^{x_{1}} d x^{\prime} \frac{w c}{a+b x^{\prime}} & =\frac{w c}{b}\left(\ln \left|a+b x_{1}\right|-\ln \left|a+b x_{0}\right|\right) \\
& =\frac{w c}{b} \ln \left|\frac{a+b x_{1}}{a+b x_{0}}\right| .
\end{aligned}
$$

Dividing this again by the normalization factor $w\left(x_{1}-x_{0}\right)$ and inserting the above parameters finally gives

$$
V_{i}^{\mathrm{av}}=\frac{L_{i} u_{i} \widehat{Q}_{i}}{\Delta N_{i}^{\max }} \ln \left|1+\frac{\Delta N_{i}^{\max }}{u_{i} \widehat{Q}_{i} \mathcal{T}_{i}^{0}}\right| \approx \frac{L_{i}}{\mathcal{T}_{i}^{0}}\left(1-\frac{\Delta N_{i}^{\max }}{2 u_{i} \widehat{Q}_{i} \mathcal{T}_{i}^{0}}\right),
$$

where we have used $\ln (1+x) \leq x-x^{2} / 2$. This formula corrects the naive formula

$$
V_{i}^{\mathrm{av}} \approx \frac{L_{i}}{\mathcal{T}_{i}}=\frac{L_{i}}{\mathcal{T}_{i}^{0}+\frac{\Delta N_{1}^{\max }}{2 u_{i} \widehat{Q}_{i}}} \approx \frac{L_{i}}{\mathcal{T}_{i}^{0}}\left(1-\frac{\Delta N_{1}^{\max }}{2 u_{i} \widehat{Q}_{i} \mathcal{T}_{i}^{0}}\right),
$$

where we have used $1 /(1+x) \approx 1-x$. Therefore, the above Taylor approximations of both formulas agree, but higher-order approximations would differ. The formulas in the main part of the paper result for $N_{i}^{\text {av }}=N_{i}^{\max } / 2$, which corresponds to the case $\delta_{i}=0$ (i.e. $f_{i}-u_{i}$ ).

Generalizing the above approach to the case $\delta_{i}>0$, we must split up the integrals into one over $w c /\left(a+b x^{\prime}\right)$ extending from $x_{0}=0$ to $x_{1}=\Delta N_{i}^{\max }$ and another one over $w c / a$ from $x_{1}=\Delta N_{i}^{\max }$ to $x_{2}=\left(1-u_{i}\right) A_{i} T^{\text {cyc }}=$ $\left(1-u_{i}\right) u_{i} \widehat{Q}_{i} T^{\mathrm{cyc}}$, where the specifications of $a, b$, and $c$ are unchanged. Taking into account $V_{i}^{0}=L_{i} / \mathcal{T}_{i}^{0}$, this gives

$$
V_{i}^{\mathrm{av}}=\frac{w L_{i} u_{i} \widehat{Q}_{i} \ln \left|1+\Delta N_{i}^{\max } /\left(u_{i} \widehat{Q}_{i} \mathcal{T}_{i}^{0}\right)\right|+Z}{w\left(1-u_{i}\right) u_{i} \widehat{Q}_{i} T^{\mathrm{cyc}}},
$$

where

$$
Z=w V_{i}^{0}\left[\left(1-u_{i}\right) u_{i} \widehat{Q}_{i} T^{\mathrm{cyc}}-\Delta N_{i}^{\max }\right] .
$$

Considering equation (15), we get

$$
V_{i}^{\mathrm{av}}=\frac{L_{i}}{\left(1-u_{i}\right) T_{\text {cyc }}} \ln \left(1+\left(1-f_{i}\right) \frac{T_{\text {cyc }}}{\mathcal{T}_{i}^{0}}\right)+V_{i}^{0} \frac{f_{i}-u_{i}}{1-u_{i}} .
$$

In second-order Taylor approximation, this results in

$$
V_{i}^{\mathrm{av}} \approx V_{i}^{0}\left[\frac{1-f_{i}}{1-u_{i}}\left(1-\frac{\left(1-f_{i}\right) T_{\mathrm{cyc}}}{2 \mathcal{T}_{i}^{0}}\right)+\frac{f_{i}-u_{i}}{1-u_{i}}\right],
$$

which can also be derived from equation (A.6), considering equation (15) and the percentage of delayed vehicles, which is given by equation (19). The same result follows from

$$
V_{i}^{\mathrm{av}}=\frac{L_{i}}{\mathcal{T}_{i}^{0}+\mathcal{T}_{i}^{\mathrm{av}}} \approx V_{i}^{0}\left(1-\frac{\mathcal{T}_{i}^{\mathrm{av}}}{\mathcal{T}_{i}^{0}}\right)
$$

together with equation (21).

\section{References}

1. D.C. Gazis, Traffic Theory (Kluwer Academic, Boston, 2002)

2. J. Esser, M. Schreckenberg, Int. J. Mod. Phys. B 8, 1025 (1997)

3. P.M. Simon, K. Nagel, Phys. Rev. E 58, 1286 (1998)

4. K. Nagel, Multi-Agent Transportation Simulations, see http://www2.tu-berlin.de/fb10/ISS/FG4/archive/ sim-archive/publications/book/

5. M. Hilliges, W. Weidlich, Transpn. Res. B 29, 407 (1995)

6. D. Helbing, J. Siegmeier, S. Lämmer, Networks and Heterogeneous Media 2, (2007)

7. M. Cremer, J. Ludwig, Math. Comput. Simul. 28, $297 \mathrm{ff}$ (1986)

8. C.F. Daganzo, Transpn. Res. B 29, 79 (1995)

9. T. Nagatani, Phys. Rev. E 48, 3290 (1993)

10. D. Chowdhury, A. Schadschneider, Phys. Rev. E 59, R1311 (1999)

11. O. Biham, A.A. Middleton, D. Levine, Phys. Rev. A 46, R6124 (1992)

12. J.-F. Zheng, Z.-Y. Gao, X.-M. Zhao, Phys. Stat. Mech. Appl. 385, 700 (2007)

13. N.A. Irwin, M. Dodd, H.G. Von Cube, Highway Research Board Bulletin 347, 258 (1961)

14. R.J. Smock, Highway Research Board Bulletin 347, 60 (1962)

15. W.W. Mosher, Highway Research Record 6, 41 (1963)

16. Bureau of Public Roads, Traffic Assignment Manual (US Dept. of Commerce, Urban Planning Division, Washington, D.C., 1964)

17. T.J. Soltmann, Highway Research Record 114, 122 (1965)

18. K.B. Davidson, in Proceedings of the $3 r d$ ARRB Conference, Part 1 (Australian Road Research Board, Melbourne, 1966), pp. 183-194 
19. R.J. Smeed, Traffic Engineering and Control 8, 455 (1966)

20. K.R. Overgaard, Traffic Quarterly, 197 (1967)

21. J.M. Thomson, Traffic Engineering and Control 8, 721 (1967)

22. J.G. Wardrop, Traffic Engineering and Control 9, 528 (1968)

23. Y. Zahavi, Traffic Engineering and Control 14, (1972)

24. R. Akcelik, Australian Road Research 21, 49 (1991)

25. K.M. Lum, H.S.L. Fan, S.H. Lam, P. Olszewski, J. Transpn. Eng. 124, 213 (1998)

26. H.M. Zhang, Transpn. Res. Rec. 1676, 109 (1999)

27. H. Tu, Monitoring Travel Time Reliability on Freeways, Ph.D. thesis, Delft University of Technology, (2008)

28. C.F. Daganzo, Transport. Res. B 41, 49 (2007)

29. N. Geroliminis, C.F. Daganzo, Transpn. Res. B 42, 759 (2008)

30. N. Geroliminis, C.F. Daganzo, Macroscopic modeling of traffic in cities, TRB 86th Annual Meeting, Paper \#07-0413, Washington D.C. (2007)

31. C.F. Daganzo, N. Geroliminis, An analytical approximation for the macroscopic fundamental diagram of urban traffic, accepted for publication (2008)

32. J.W. Godfrey, Traffic Engineering and Control 11, 323 (1969)
33. R. Herman, I. Prigogine, Science 204, 148 (1979)

34. R. Herman, S. Ardekani, Transportation Science 18, 101 (1984)

35. M. Eichler, C.F. Daganzo, Transportation Research B 40, $731(2006)$

36. D. Helbing, Rev. Mod. Phys. 73, 1067 (2001)

37. D. Helbing, M. Treiber, A. Kesting, M. Schönhof, Theoretical vs. empirical classification and prediction of congested traffic states, Eur. Phys. J. B, accepted for publication (2009), see e-print http://arxiv.org/abs/0903.0929

38. G.B. Whitham, Linear and Nonlinear Waves (Wiley, New York, 1974)

39. R. Hall, Queueing Methods for Service and Manufacturing (Prentice Hall, Upper Saddle River, NJ, 1991)

40. D. Helbing, J. Phys. A: Math. Gen 36, L593 (2003)

41. D. Helbing, A. Mazloumian, Operation regimes and sloweris-faster effect in the control of traffic intersections, submitted (2008), see e-print

http://arxiv.org/abs/0903.0926

42. D. Helbing, T. Seidel, S. Lämmer, K. Peters, in Econophysics and Sociophysics, edited by B.K. Chakrabarti, A. Chakraborti, A. Chatterjee (Wiley, Weinheim, 2006), p. 552 